\title{
Development of a computer aided product-form design tool based on numerical definition scheme and neural network
}

\author{
Hung-Yuan CHEN* and Yu-Ming CHANG** \\ * Department of Visual Communication Design, Southern Taiwan University of Science and Technology \\ No. 1, Nantai Street, Yongkang District, Tainan City 71005, Taiwan, ROC \\ E-mail: hungyuan@mail.stust.edu.tw \\ ** Department of Creative Product Design, Southern Taiwan University of Science and Technology \\ No. 1, Nantai Street, Yongkang District, Tainan City 71005, Taiwan, ROC
}

Received 23 March 2014

\begin{abstract}
The evolution of a product form during the design process is typically governed by the designer's individual preferences and creative instincts. As a consequence, there is a risk that the product form may fail to satisfy the consumers' expectations or may induce an unanticipated consumer response. This study commences developing a computer aided product-form design (CAPD) tool based on numerical definition scheme (NDS) and back-propagation neural network (BPNN). A NDS approach is employed to depict an explicit product form, and a series of evaluation trials are then performed to establish the correlation between the product form features and the consumers' perceptions of the product image. The results of the evaluation trials are used to construct a BPNN model to predict the likely consumer response to any arbitrary product form. The feasibility of developing a CAPD tool is demonstrated using a 3D knife form in this study.
\end{abstract}

Key words : Product form, Consumers’ perception, CAPD, NDS, BPNN

\section{Introduction}

\subsection{Background and Motivation}

Modern day life is characterized by accelerating technological change. Similarly, the field of product design is being rapidly transformed through the introduction of new materials, advanced technologies, sophisticated information-processing techniques, and so forth. Therefore, reducing the lead-time of the product development stages is a key consideration for many enterprises. Although advanced technologies have accelerated the use of computers in performing the computer-aided design (CAD) assignments during the product development, generally speaking, these assignments involve product's configuration, product's functionality and fitness for use. These CAD product developments only address the tangible functional aspects of a product. Recent design trends show an inclination towards objects which inspire consumers, enhance their lives, and help in triggering emotions. Product design directed by consumers' emotional content or psychological perception can be regarded as the heart of current product design practices and research (Demirbilek and Sener, 2003; McDonagh and Denton, 2005). Accordingly, in designing a product to meet consumers' expectations, a designer should consider not only the tangible functional aspects of a product, but also the intangible psychological perception of a product. However, product design activities are often reduced to a discussion based on the designers' opinions. Although many of the design assignments performed by product designers at the conceptual design stage involve the consideration of the potential consumers' perception of the product form, few computer-aided design tools are available to support this specific set of design activities. To objectively develop a specific computer-aided design tool, many systematic design approaches have been proposed for modeling the correlation between the form features of a product and the consumers' psychological perception of the image projected by the product (Nagamachi, 2002; Choi and Jun, 2007; Salhieh, 2007; Huang et al, 2011; Huang et al, 2012). Of these systematic design approaches, Kansei Engineering (KE) is a fundamental consumer-oriented systematic design approach in which the consumers' feelings or product image perceptions are expressed using suitable image 
descriptors (Nagamachi, 2002) and it has emerged as one of the most powerful techniques for taking explicit account of the correlation between the product form and its projected image. However, the effectiveness of developing a specific computer-aided design tool based on the KE approaches is crucially determined by the definition of the design features used to describe the product details and the choice of analytical method used to analyze the correlation between the product form and the corresponding consumer response.

\subsection{Definition of Design Features Used to Describe Product Form}

Traditional KE approaches proposed in the literature (Nagamachi, 2002; Han and Hong, 2003; Lai et al, 2005; Van Lottum et al, 2006; Wang, 2011) have a fundamental weakness in defining a product form or its features in that they rely on the use of qualitative concepts such as "items / factors" and "categories / levels" based on pictorial profiles with accompanying text descriptions to generate high-level descriptions of the design variables which collectively define the overall product form. Figure 1 illustrates the application of such a technique to the case of a mobile phone. As shown, the product form of the phone is defined in terms of three items, namely "top shape", "body shape", and "bottom shape", respectively, with each item having three or four different categories / levels. However, such methods only allow general variations in the product form to be expressed and thus their application is limited to the conceptual design stage. Moreover, since such techniques only permit a modeling of the high-level product form features, they can not be used as the basis for evaluation schemes intended to assess the effects of detailed product form changes on the consumers' perceptions of the product's projected image. In other words, when such qualitative methods are employed, the services of an experienced designer are still required to subjectively conjecture the likely consumer response to subtle changes in the product design. Therefore, an outstanding requirement exists for product form definition methods capable not only of capturing and expressing subtle variations in the product form, but also of modeling the changes induced in the consumers' psychological response to the product by these product form changes. The numerical definition approach, in which the product form is described in terms of the control point positions of mathematical curves expressed in coordinate dimensions, provides a more suitable means of defining product form. NURBS (non-uniform rational B-splines) are the most popular mathematical model curves for developing computer software (e.g. Rhinoceros 3D based on NURBS curves). These curves are very efficient and convenient to construct, and are therefore used by the present study to define the product form. Adopting this numerical definition approach has the advantage that detailed variations of the product form can be explicitly depicted. Moreover, its use facilitates the subsequent development of a computer aided product-form design (CAPD) tool in this study.

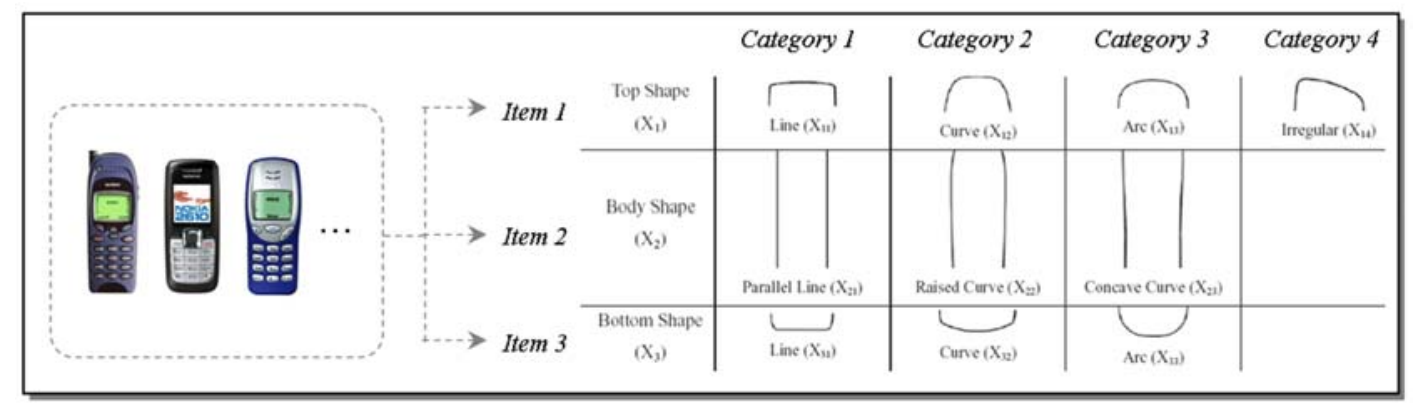

Fig. 1 Application of “items/factors” and “categories/levels” to example of mobile phone

\subsection{Analytical Techniques for Constructing Product Form-Consumer Perception Models}

In choosing a suitable analytical technique with which to model the relationship between the product form and the consumer response, conventional statistical analysis techniques such as Multiple Regression Analysis (MRA), Conjoint Analysis (Green and Srinivasan, 1978) and Quantitative Theory Type I (Hayashi, 1976) are commonly employed to interpret the relationships between the independent and dependent variables. However, the accuracy of the predicted results is seriously degraded if the independent and dependent variables are characterized by a non-linear relationship (Weiss, 1983) or if a multi-collinearity effect exists between the two sets of variables. In contrast to the conventional statistical analysis techniques, back-propagation neural network (BPNN) method is free of the restriction on the type of relationship between the independent variables and dependent variables when constructing prediction models. Figure 2 shows a typical three-layered BPNN with an input layer, an output layer and a single hidden layer. In this figure, $n, m$ 
and $p$ represent the number of neurons in the input layer, output layer and hidden layer, respectively (indexed by $i, j$, and $k$, respectively). Meanwhile, $w_{i j}$ and $w_{j k}$ represent the weights of the connections between neuron $i(i=1,2, \ldots, n)$ and neuron $j(j=1,2, \ldots, m)$, and between neuron $j(j=1,2, \ldots, m)$ and neuron $k(k=1,2, \ldots, p)$, respectively. In training this network, a set of input patterns or signals, $\left(x_{1}, x_{2}, \ldots, x_{n}\right)$, is presented to the network input layer. The network then propagates the inputs from layer to layer and provides the output at the neurons of the output layer (Negnevitsky, 2002). In an iterative process, the output results are compared to the required values, and the resultant error signals propagated back through the BPNN layers. The weights of the individual network connections are progressively adjusted until the specified error criterion is satisfied. During the verification and subsequent operation of the BPNN, the network generates an output by applying the weights established during the training process to the input variables as they are propagated from layer to layer. With their effective learning, storage and prediction capabilities, BPNN provide a powerful means of examining the complex relationships between input variables (i.e. the product form definition) and output variables (i.e. the consumers' image perception). Consequently, comparing the relative merits of conventional linear analysis techniques with that of non-linear BPNN analysis techniques, it seems that the latter are better suited to data analysis applications since they are applicable both to data sets with linear patterns and to those with nonlinear patterns.

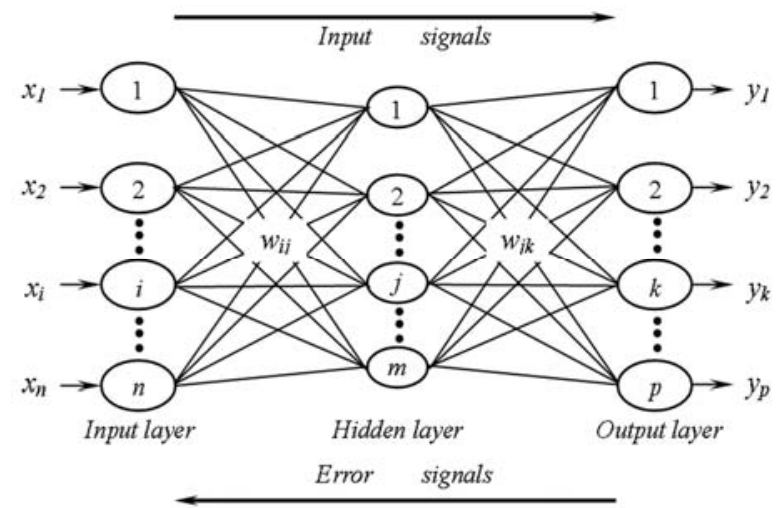

Fig. 2 Three-layered BPNN structure

\subsection{Objective and Overview of Implementation Procedure}

This study commences by developing a CAPD prototype tool in which a numerical definition of the product form is supplied to a trained BPNN, which then responds with the corresponding consumer image perception. The feasibility of developing a CAPD tool is demonstrated using a knife form for illustration purposes. This product is chosen quite specifically here since knives have broadly similar functional structures, and hence the consumer response is governed primarily by the design and appearance of the knife rather than its functional capabilities. A numerical definition scheme (NDS) is employed to construct an explicit product form depiction by synthesizing the size, angle, proportion and coordinate features of a knife form. The resulting knife form definition is then used to conduct an experimental study to investigate the consumers' psychological perception of the image projected by the knife form. The relationship between the consumers' image perception of a knife form and the corresponding product form design is modeled using the BPNN. Using the trained BPNN model as its basis, a CAPD tool is created using Visual Basic software. The performance of the developed tool is verified by comparing its predictions for the image perception of six knife forms with the image perception results obtained experimentally.

\section{Product Form Numerical Definition and Evaluation Samples 2.1 Numerical Definition Scheme (NDS) of Knife Form}

Having conducted an extensive review of commercially-available knives, a generic knife form was defined comprising two basic components, i.e. a blade and a handle. The blade profile was described using two curves, namely one curve to describe the contour of the upper edge of the blade and a second curve to describe the profile of the lower, cutting edge. To support the modeling of detailed variations in the curve profiles, the two curves were defined as NURBS curves, and were constructed using a total of seven control points (i.e. p1 p7). As shown in Figure 3, control point $\mathrm{p} 1$, located at the intersection point of the upper and lower curves was specified as the origin of the coordinate 
system used to describe the $\mathrm{X}$ - and Y-axis coordinate locations of each control point in the overall knife form definition. Variations in the form of the knife handle were modeled using six adjustable design elements, namely a centric curve to define the contour of the lower edge of the handle, a width-to-height proportion parameter, a sectional area proportion parameter, a rounded radius parameter, a sectional inclination parameter, and a tail arc curve. As shown in Figure 3, the centric curve representing the central backbone of the knife handle was modeled using a three-degree NURBS curve with five control points (p8 p12). To facilitate the introduction of detailed variations in the form of the knife handle, five section lines were uniformly distributed along its length; forming the fore-section, the 2nd section, the 3rd section, the 4th section and the tail-section, respectively.

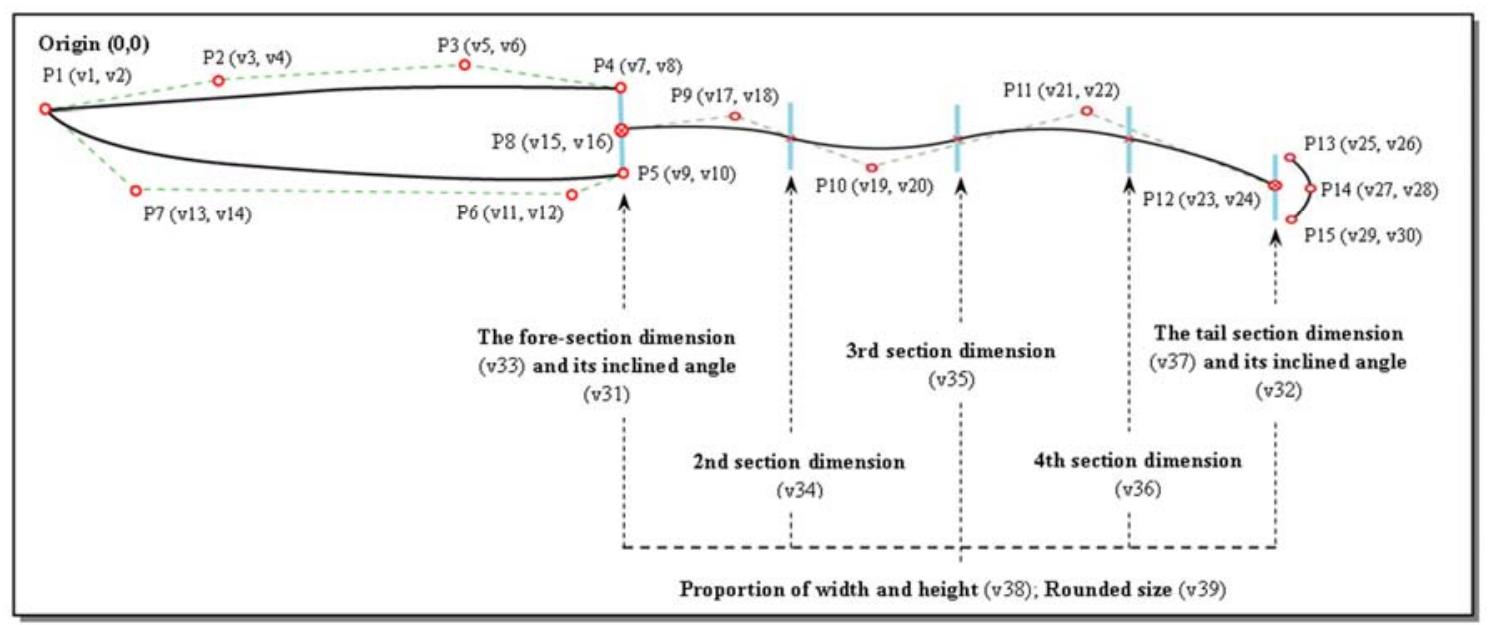

Fig. 3 Complete numerical definition of generic knife form

As shown in Figure 4(a), each lateral section of the knife handle is initially assigned a square cross-section with default dimensions of $20 \times 20 \mathrm{~mm}$. During the design process, however, detailed variations in the handle form are introduced by specifying appropriate values of the width-to-height parameter (i.e. $1,0.67$ or 0.5 ), the sectional area proportion parameter (i.e. 1, 0.56 or 0.25 ), and the rounded radius parameter (i.e. $1 \mathrm{~mm}, 3 \mathrm{~mm}$ or $5 \mathrm{~mm}$ ). Note that in modifying the default form of the handle, the various sections can be assigned different values of the sectional area proportion parameter, but must have the same width-to-height and rounded radius settings. As shown in Figure 4(b), further variations in the handle form can be introduced by specifying appropriate values of the sectional inclination parameter (i.e. $60^{\circ}, 90^{\circ}$ or $120^{\circ}$ ) for the fore- and tail-sections of the handle, respectively. Finally, detailed variations in the shape of the tail-section are accomplished by adjusting the positions of the three control points p13 p15. The thirty-nine design variables which together constitute the explicit numerical definition of the knife form are defined in Table 1.

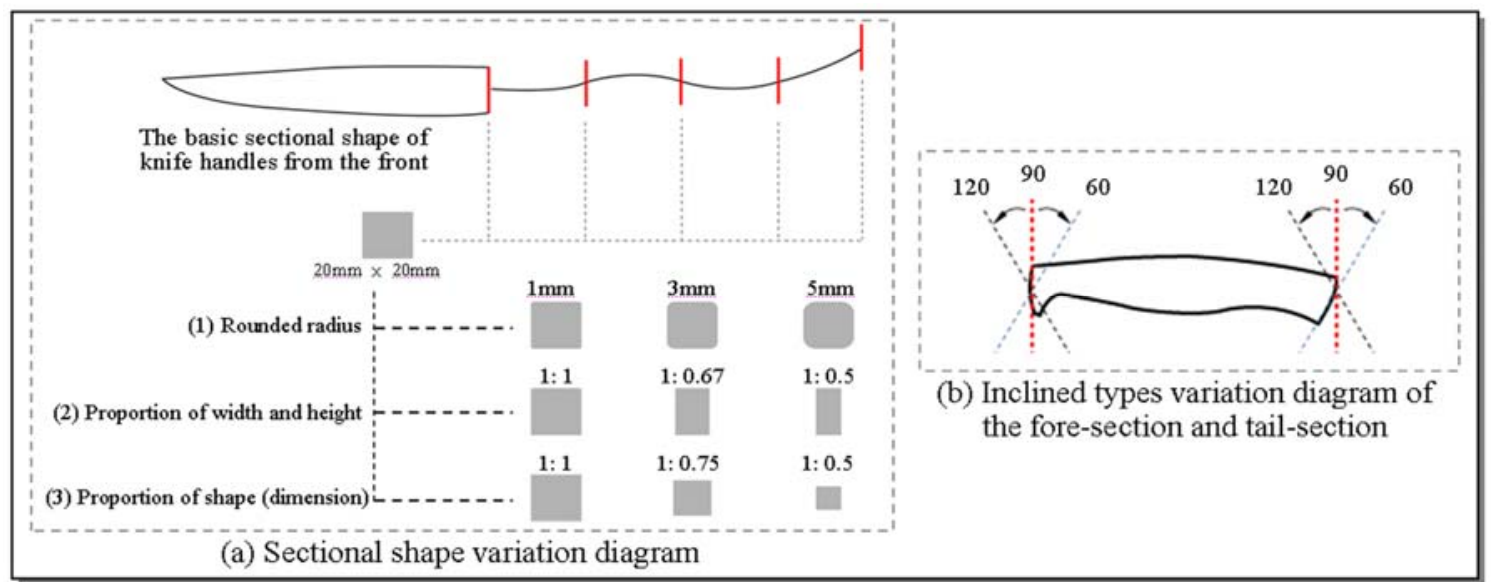

Fig. 4 The variation diagram of sectional shape (a), and the fore-section and tail-section inclined types (b) for knife handle 
Table 1. Numerical variables used to create explicit definition of knife design

\begin{tabular}{|c|c|c|c|c|}
\hline \multicolumn{3}{|c|}{ Knife form decomposed descriptions } & Variables specification & Variables \\
\hline \multirow{2}{*}{ Knife blade } & \multicolumn{2}{|c|}{ Curve of blade back } & Coordinate data of 4 control points $\left(\mathrm{p}_{1} \sim \mathrm{p}_{4}\right)$ & $\mathrm{v} 1 \sim \mathrm{v} 8$ \\
\hline & \multicolumn{2}{|c|}{ Curve of blade edge } & Coordinate data of 3 control points $\left(\mathrm{p}_{5} \sim \mathrm{p}_{7}\right)$ & $\mathrm{v} 9 \sim \mathrm{v} 14$ \\
\hline \multirow{11}{*}{ Knife handle } & \multicolumn{2}{|c|}{ Centric curve of knife handle } & Coordinate data of 5 control points $\left(\mathrm{p}_{8} \sim \mathrm{p}_{12}\right)$ & $\mathrm{v} 15 \sim \mathrm{v} 24$ \\
\hline & \multicolumn{2}{|c|}{ Tail arc curve of knife handle } & Coordinate data of 3 control points $\left(\mathrm{p}_{13} \sim \mathrm{p}_{15}\right)$ & v25 v30 \\
\hline & \multirow{9}{*}{$\begin{array}{c}\text { Sectional } \\
\text { detail } \\
\text { variation of } \\
\text { knife handle }\end{array}$} & Fore-section inclination of knife handle & Inclination $\left(60^{\circ}, 90^{\circ}, 120^{\circ}\right)$ & v31 \\
\hline & & Tail-section inclination of knife handle & Inclination $\left(60^{\circ}, 90^{\circ}, 120^{\circ}\right)$ & v32 \\
\hline & & Fore-section shape dimension & Proportion of shape dimension $(1,0.75,0.5)$ & v33 \\
\hline & & 2nd section shape dimension & Proportion of shape dimension $(1,0.75,0.5)$ & v34 \\
\hline & & 3rd section shape dimension & Proportion of shape dimension $(1,0.75,0.5)$ & v35 \\
\hline & & 4th section shape dimension & Proportion of shape dimension $(1,0.75,0.5)$ & v36 \\
\hline & & Tail-section shape dimension & Proportion of shape dimension $(1,0.75,0.5)$ & v37 \\
\hline & & Proportion of width and height on all sections & Dimensional proportion of width / height $(1,0.67,0.5)$ & v38 \\
\hline & & Rounded radius of all sections & Rounded radius (1mm, 3mm, 5mm) & v39 \\
\hline
\end{tabular}

\subsection{Creating 3D Knife Models}

To create 3D knife models of image evaluation, three industrial designers, each with more than three years' of product form design experience were invited to create 3D knife form models using the numerical definition scheme (NDS). Having completed each design, the corresponding data set was recorded in a design database. A total of forty-three knife models were created using Rhinoceros software, and these models were identically rendered using a quasi-gray material and were presented in an identical pose under identical lighting conditions.

\subsection{Defining Variation of Each 3D Knife Model from the Criterion Knife Form}

Since the numerical data sets corresponding to the forty-three 3D knife models all vary in terms of their coordinate data, inclination angles, dimensional proportions and rounded radius values, respectively, it is difficult to interpret the analytical results regarding the relationship between detailed variations in the knife form and the corresponding change in the consumers' emotional response to the product image. Consequently, an average 3D knife form was generated using a shape averaging method (Chen and Parent, 1989) to create a criterion against which to define the variation of each knife form. As shown in Figure 5, the criterion (i.e. mean) knife form was created by averaging the design variables of the forty-three knife form models. The variation of the design variables of each of the forty-three knife form models from the corresponding design variables of criterion knife form models was then computed in order to create a relative numerical data set for each model to provide the independent variables (i.e. the design variables) used in the subsequent analysis of the correlation between the knife form and the corresponding product image.

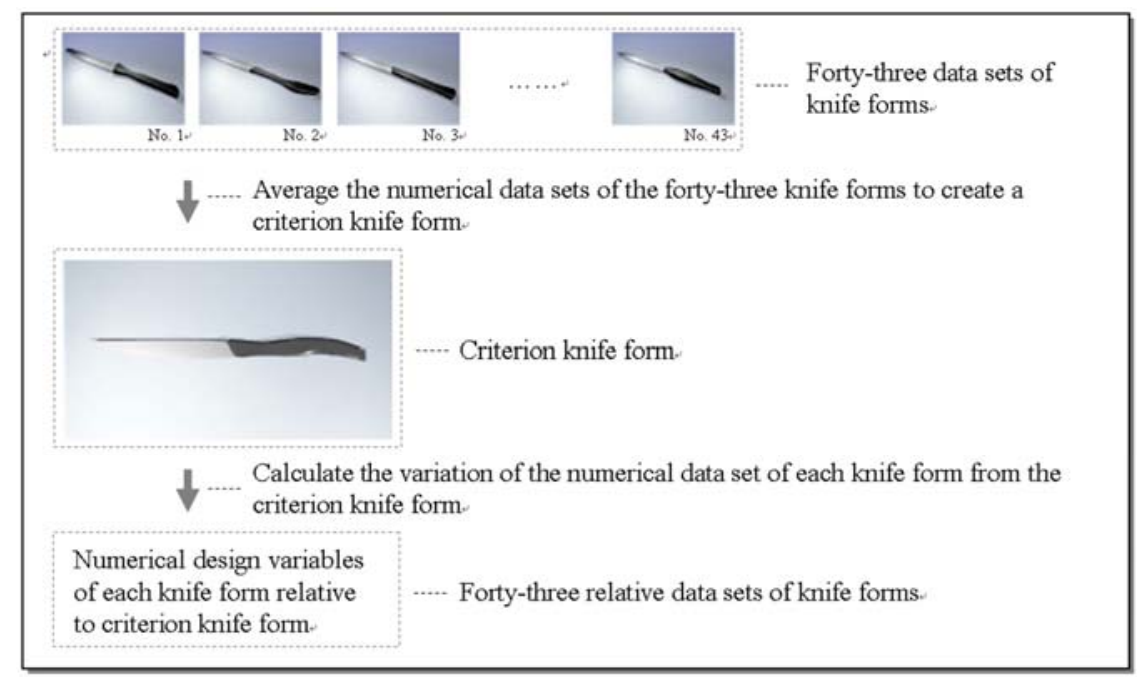

Fig. 5 Defining variation of forty-three knife forms from the criterion knife form

\subsection{Adjustable Variation Range of Each Design Variable}

The numerical data sets corresponding to the forty-three 3D knife models comprise inclination angles, dimensional proportions, rounded radius values and coordinate data, respectively. In addition to the coordinate data, other types of 
numerical data sets have definite range of detailed variation. To further make clear range of coordinate data, the maximum coordinate variation (distance) and the minimum coordinate variation (distance) of design variables of each of the forty-three knife models from the coordinate value of corresponding design variables of criterion knife form were considered as the adjustable positive range (i.e. rightward for X-axis and upward for Y-axis) and the adjustable negative range (i.e. leftward for X-axis and downward for Y-axis) for each design variable, respectively. Table 2 shows the adjustable range of each design variable using a unit of millimeter.

Table 2. Adjustable variation range of each design variable for knife form

\begin{tabular}{|c|c|c|c|c|c|c|c|c|c|c|}
\hline & $\mathrm{v} 1$ & $\mathrm{v} 2$ & v3 & $\mathrm{v} 4$ & v5 & v6 & v7 & v8 & v9 & v10 \\
\hline Adjustable positive range (distance) & 0.00 & 0.00 & 19.71 & 16.31 & 20.32 & 15.47 & 4.72 & 12.38 & 5.27 & 12.88 \\
\hline Criterion knife form & 0.00 & 0.00 & 18.62 & 10.38 & 83.41 & 8.60 & 119.72 & 8.37 & 120.27 & -5.42 \\
\hline \multirow[t]{2}{*}{ Adjustable negative range (distance) } & 0.00 & 0.00 & 24.50 & 11.76 & 21.99 & 12.76 & 5.28 & 10.64 & 4.83 & 9.60 \\
\hline & v11 & $\mathrm{v} 12$ & $\mathrm{v} 13$ & v14 & v15 & $\mathrm{v} 16$ & v17 & $\mathrm{v} 18$ & v19 & v20 \\
\hline Adjustable positive range (distance) & 24.51 & 13.67 & 10.20 & 14.50 & 15.12 & 10.40 & 0.11 & 15.05 & 3.17 & 13.93 \\
\hline Criterion knife form & 88.60 & -6.13 & 19.05 & -7.50 & 115.13 & 1.42 & 150.01 & 2.15 & 180.07 & 0.47 \\
\hline Adjustable positive range (distance) & 0.73 & 16.35 & 4.11 & 21.86 & 5.13 & 20.00 & 9.54 & 23.51 & 5.01 & 13.73 \\
\hline Criterion knife form & 210.03 & 1.85 & 240.01 & 1.36 & 240.13 & 8.20 & 249.54 & 0.61 & 239.91 & -5.47 \\
\hline \multirow[t]{2}{*}{ Adjustable negative range (distance) } & 1.97 & 25.63 & 6.09 & 14.59 & 4.87 & 14.29 & 12.06 & 23.97 & 5.19 & 15.00 \\
\hline & v31 & v32 & v33 & v34 & v35 & v36 & v37 & v38 & v39 & \\
\hline Adjustable positive range & $30^{\circ}$ & $30^{\circ}$ & 0.44 & 0.44 & 0.44 & 0.44 & 0.44 & 0.33 & $2 \mathrm{~mm}$ & \\
\hline
\end{tabular}

The unit of distance is millimeter.

\section{Selection of Product Image Descriptors}

The adjectives used by consumers when expressing their perceptions of a particular product provide an explicit representation of their abstract emotional response to the product form, and therefore furnish designers with valuable clues regarding the consumers' product image expectations and the likelihood of the success of the generated product form in meeting its design objectives. In this study, around one-hundred-and-fifty adjectives (in Chinese) pertaining to knives of various types were collected from web sites, magazines, catalogues and so forth. These adjectives were sieved by the three industrial designers in accordance with the criteria outlined below such that just thirty-one adjectives remained.

- Criterion 1: retain the adjective only if it relates specifically and entirely to the product form. In other words, any adjectives relating either directly or indirectly to the product's color for example (e.g. bright, vivid, colorful...) should be rejected.

- Criterion 2: retain the adjective only if it is subject to common usage. In other words, any adjectives which are infrequently used or which are liable to introduce confusion and/or uncertainty should be rejected.

However, if the all thirty-one product image descriptors were directly used to commence the sequent "knife form image evaluation", the loading of knife form image evaluation would be heavy for each subject since they would have to perform the 1333 (43 knives $\times 31$ descriptors) times of knife form image evaluation. Therefore, it was necessary to objectively select the representative product image descriptors so as to alleviate the loading of knife form evaluation for each subject. To the selection of representative product image descriptors, a multi-step procedure involving the combination of Kawakida Jirou (K.J.) classification activity and the hierarchical clustering technique, and a questionnaire-based statistical analysis process was performed to select a small number of these thirty-one adjectives as product image descriptors with which to categorize the potential product image perceptions induced by the forty-three knife form models. The details of multi-step procedure are described in the sections below.

\subsection{Select Representative Knife Models}

To simplify the process of identifying suitable product image descriptors whilst simultaneously ensuring the ability of these descriptors to represent the characteristics of all the knife forms, an initial classification procedure was performed to identify a small number of representative knife form models amongst the total set of forty-three models. 
The 3D knife models were individually displayed on A5-sized paper cards and sixteen subjects (eight male and eight female; average age twenty-seven; eleven with design backgrounds and five with non-design backgrounds) were invited to participate in a K.J. sorting and classification procedure (see Step 1 below). Due to the subjective judgments of K.J. classification, a statistical-based approach (i.e. hierarchical clustering technique based on Ward's method (Ward, 1963)) was then introduced to objectively analyze the K.J. classification results obtained from the sixteen subjects such that the deficiency of the subjective judgment using K.J. approach can be improved and the representative knife form models can be objectively selected. Representative knife form models were then selected using a step-by-step statistical-based clustering approach, as described in the following:

Step 1: Using the K.J. approach, the paper cards were sorted into 5-10 groups based on the perceived degree of similarity between the individual 3D knife forms.

Step 2: A similarity matrix was constructed based on the classification results obtained in Step 1.

Step 3: The similarity matrix was transformed into a corresponding dissimilarity matrix.

Step 4: A hierarchical clustering technique based on Ward's method was applied to the dissimilarity matrix data to generate a cluster tree diagram.

Step 5: Knife model groups were determined from an inspection of the clustering results.

Step 6: The center of each knife model group was calculated and the Euclidean distance of each knife model relative to its respective group center was computed.

Step 7: Representative knife models were selected by identifying the model in each group with the minimum Euclidean distance.

Figure 6 shows the members of each of the five knife model groups identified using this step-by-step approach.

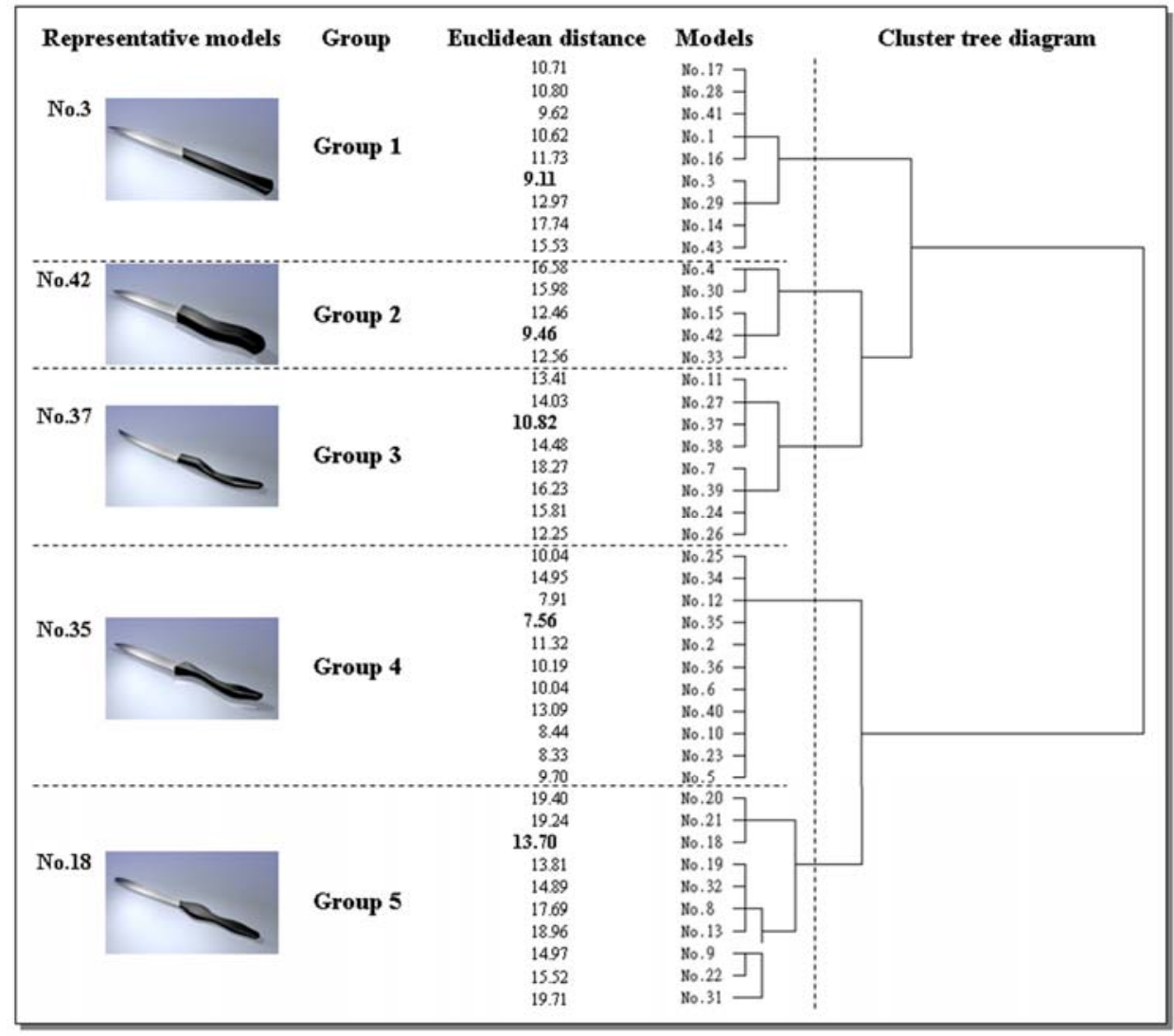

Fig. 6 Representative 3D knife models

\subsection{Selection of Representative Product Image Descriptors}

The five representative models and thirty-one potential product image descriptors were integrated into a questionnaire type document, in which nine-point Likert scales with anchors ranging from "strongly disagree (1)” to "strongly agree (9)" were used to evaluate the extent to which the subjects felt that each of the image descriptors described the emotional response invoked by the five models. The sixteen subjects who participated in the K.J. 
classification procedure were then asked to complete the questionnaire. The evaluation data provided by each subject were compiled and a process of factor analysis using principal component analysis as an extraction technique and varimax as an orthogonal rotation method was conducted to simplify the factor structure of the thirty-one product image adjectives. A minimum eigenvalue of 1 was then used as the cutoff value for extraction purposes. Table 3 presents the simplified factor structure of the thirty-one product image adjectives and indicates the corresponding factor loadings. As shown, the adjectives are classified into three main factors, accounting collectively for $91.9 \%$ of the cumulative variance explained.

Factor 1, which accounts for 37.5\% of the variance, includes such product image adjectives as "individualized", “masculine”, “vital”, “peculiar”, “vivid”, “innovative”, “spirited”, and so on. These adjectives relate in the main to the personality, singularity and dynamic characteristics of the product. As shown, the "individualized” adjective accounts for $98.3 \%$ of the variance explained in this factor. Therefore, Factor 1 can be interpreted as a "personality factor" and the "individualized" adjective selected as a suitable product image descriptor to describe the majority of the image adjectives within this factor. Factor 2, which accounts for $32.9 \%$ of the variance, includes adjectives such as “elegant”, "tasteful”, "solemn”, "luxurious”, “posh”, "high-class”, and so forth. The majority of the adjectives in this factor relate to the aesthetic properties of the knife form. It is observed that the "elegant" adjective accounts for $95.4 \%$ of the variance explained in this factor. Therefore, Factor 2 can be interpreted as an "aesthetic factor" and the "elegant" adjective chosen as a representative product image descriptor. Finally, Factor 3, which accounts for 21.5\% of the variance, includes such adjectives as “modern”, “traditional”, “concise”, “dapper”, “futuristic” and “common”, which all relate to the time and trend attributes of the knife model. From inspection, it is found that the "modern" adjective accounts for $96.9 \%$ of the variance explained by this particular factor. Accordingly, Factor 3 is designated as the "trend factor" and is represented using the "modern” descriptor.

Table 3. Simplified factor structure of 31 product image adjectives

\begin{tabular}{|c|c|c|c|}
\hline Product Image Adjective & Factor 1 & Factor 2 & Factor 3 \\
\hline Individualized* & 0.983 & -0.172 & -0.033 \\
\hline Masculine & -0.976 & 0.066 & -0.075 \\
\hline Vital & -0.947 & -0.238 & -0.192 \\
\hline Peculiar & -0.914 & 0.320 & -0.086 \\
\hline Innovative & 0.884 & -0.241 & -0.384 \\
\hline Spirited & 0.871 & -0.275 & -0.184 \\
\hline Unconstrained & 0.861 & -0.406 & -0.108 \\
\hline Popular & 0.841 & -0.119 & -0.185 \\
\hline Convergent & 0.806 & 0.195 & 0.259 \\
\hline Unique & -0.775 & 0.288 & 0.199 \\
\hline Dynamic & 0.747 & -0.215 & -0.211 \\
\hline Simple & 0.645 & 0.435 & 0.296 \\
\hline Conservative & 0.616 & 0.370 & -0.257 \\
\hline Newfangled & 0.499 & 0.468 & 0.346 \\
\hline Elegant* & -0.161 & 0.954 & 0.213 \\
\hline Tasteful & -0.275 & 0.937 & 0.117 \\
\hline Solemn & -0.303 & 0.937 & -0.158 \\
\hline Luxurious & 0.128 & 0.925 & 0.358 \\
\hline Posh & 0.406 & -0.902 & -0.075 \\
\hline High-class & 0.349 & -0.859 & 0.375 \\
\hline Elaborate & 0.252 & 0.828 & -0.014 \\
\hline Delight & 0.222 & -0.759 & -0.309 \\
\hline Specialized & 0.188 & 0.756 & 0.245 \\
\hline Refined & -0.221 & 0.744 & 0.329 \\
\hline Staid & -0.206 & 0.635 & 0.274 \\
\hline Modern* & 0.116 & -0.205 & 0.969 \\
\hline Traditional & -0.157 & 0.323 & -0.787 \\
\hline Concise & -0.231 & 0.200 & 0.730 \\
\hline Dapper & -0.364 & 0.305 & 0.708 \\
\hline Futuristic & -0.346 & 0.334 & 0.671 \\
\hline Common & -0.309 & 0.441 & 0.645 \\
\hline Eigenvalue & 15.09 & 8.69 & 2.49 \\
\hline Variance explained & $37.5 \%$ & $32.9 \%$ & $21.5 \%$ \\
\hline Cumulative variance explained & $37.5 \%$ & $70.4 \%$ & $91.9 \%$ \\
\hline
\end{tabular}

Asterisks indicate the representative image description 


\section{Evaluation of Knife Form Image}

Fifty-six subjects (thirty-one male, twenty-five female; aged between twenty-three and thirty-five) were invited to evaluate the product images projected by the forty-three 3D knife models. In the evaluation procedure, the emotional responses induced by each model were quantified using three Likert scales, i.e. one scale for each of the three product image descriptors identified in Table 3. In accordance with the $7 \pm 2$ limit on an individual's capacity for processing information (Miller, 1983), each scale was constructed with nine points. As shown in Figure 7, the forty-three knife form samples and the three Likert scales were integrated into an evaluation interface constructed using Visual Basic software. After each subject had evaluated all of the samples, the evaluation data were recorded for analysis purposes.

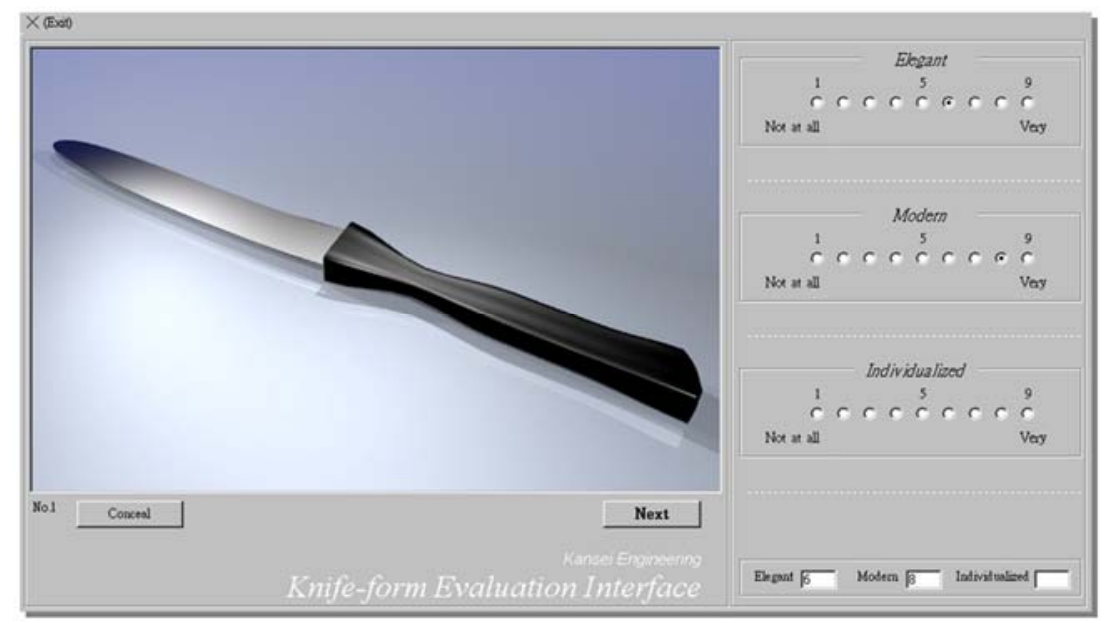

Fig. 7 Knife form evaluation interface

\section{Establishing the Relationship between Knife Form and Consumer's Image Perception using BPNN}

In this study, the image evaluation results obtained for the 3D knife forms were analyzed using a back-propagation neural network (BPNN) model. Mechanizing the relationship between the specific knife form and the corresponding image perception was performed in three steps: (1) establishing a suitable BPNN model, (2) training the BPNN model and (3) verifying the performance of the BPNN model. In attempting to define an optimal BPNN model, three models differed only in terms of the number of neurons in their single hidden layer. These BPNN models were constructed, in which the number of neurons in the single hidden layer was determined using the Arithmetic mean (Arith.), Geometric mean (Geom.), and Sum (Sum) configuration rules, respectively. The configurations of each of the three BPNN models are summarized in Table 4. In constructing and training the BPNN models to predict the likely consumer response to any 3D knife form designed using the NDS, the thirty-nine design variables for each knife form were supplied to the input neurons of the BPNN, while the average values of the three product image evaluations obtained in the manual evaluation procedure were used as the target values for the output neurons.

Table 4. Number and arrangement of neurons in three knife form BPNN models

\begin{tabular}{ll}
\hline BPNN model & Number of neurons in each layer \\
\hline \multirow{3}{*}{ BPNN-Arith. } & Input layer: 39 neurons for 39 design variables \\
& Hidden layer: 21 neurons, $(39+3) / 2=21$ \\
& Output layer: 3 neurons for values of 3 image descriptors \\
& Input layer: 39 neurons for 39 design variables \\
BPNN-Geom. & Hidden layer: 11 neurons, $(39 \times 3) \wedge 0.5=10.8 \doteqdot 11$ \\
& Output layer: 3 neurons for values of 3 image descriptors \\
& Input layer: 39 neurons for 39 design variables \\
& Hidden layer: 42 neurons, $(39+3)=42$ \\
& Output layer: 3 neurons for values of 3 image descriptors \\
\hline
\end{tabular}


The BPNN models were trained using the numerical definition data of the forty-three knife form samples and the corresponding image perception values established using the manual evaluation procedure. The training process was performed using a sigmoid transformation function and the delta-rule training rule, respectively. It was found that in all three BPNN models, the RMSE fell to a value of less than 0.08 after approximately 2000 epochs. The full set of training results are summarized in Table 5. It is observed that for each model, the minimum RMSE value is obtained after 80,000 training epochs. In general, the three models provide a similar level of predictive accuracy, i.e. the difference in the three RMSE values is less than $0.005(0.0256-0.0212=0.0044)$. From inspection, it is determined that the BPNN-Arith. model yields the lowest prediction errors of the three models, i.e. an RMSE value of 0.0212 after 80,000 epochs. Consequently, the BPNN-Arith. model (with the neuron weights obtained after 80,000 training epochs) was adopted as the operational BPNN model for predicting the knife form image values associated with any given numerical knife form definition.

Table 5. RMSE of BPNN models for knife form training dataset

\begin{tabular}{cccc}
\hline Number of training epochs & BPNN-Arith. & BPNN-Geom. & BPNN-Sum \\
\hline 1000 & 0.0785 & 0.0707 & 0.0722 \\
2000 & 0.0707 & 0.0600 & 0.0617 \\
3000 & 0.0678 & 0.0572 & 0.0583 \\
5000 & 0.0619 & 0.0539 & 0.0554 \\
10,000 & 0.0446 & 0.0486 & 0.0501 \\
150,00 & 0.0377 & 0.0411 & 0.0427 \\
20,000 & 0.0331 & 0.0371 & 0.0401 \\
30,000 & 0.0269 & 0.0293 & 0.0334 \\
40,000 & 0.0243 & 0.0268 & 0.0299 \\
50,000 & 0.0234 & 0.0256 & 0.0286 \\
60,000 & 0.0225 & 0.0245 & 0.0273 \\
70,000 & 0.0216 & 0.0234 & 0.0261 \\
80,000 & $\mathbf{0 . 0 2 1 2}$ & $\mathbf{0 . 0 2 2 9}$ & $\mathbf{0 . 0 2 5 6}$ \\
90,000 & 0.0212 & 0.0229 & 0.0256 \\
\hline
\end{tabular}

To verify the predictive ability of the functional BPNN-Arith. model, six new knife forms were designed using the respective NDS schemes, as shown in Figure 8, respectively. In the verification process, the six knife form samples were input to the evaluative interface shown in Figure 7 and were evaluated by a second group of thirty subjects (seventeen male, thirteen female; aged between twenty-three and thirty-five years old) using three nine-point Likert scales. The product image perception in each of the three domains associated with the 3D knife form were also predicted by substituting the relevant design variable values of each verification sample into the functional models, respectively. The discrepancy between the Likert scale evaluation scores assigned to each verification sample by the two groups of subjects and those predicted by the prediction models was assessed using the root-mean-square-error index (abbreviated for convenience hereafter to EI) given in Eq. (1).

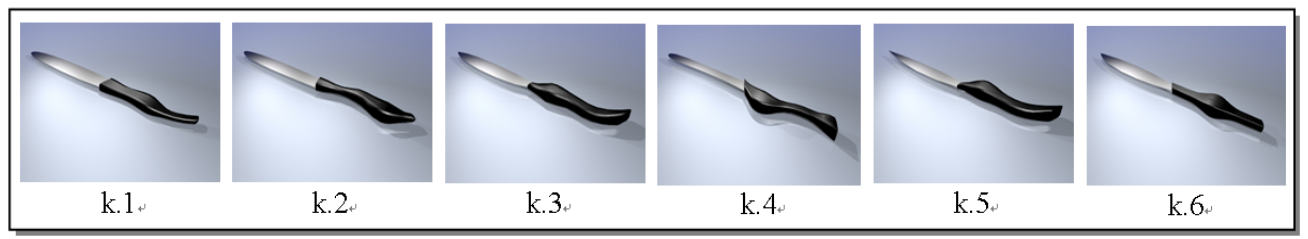

Fig. 8 Knife form models used for functional model verification purposes

$$
\mathrm{EI}=\sqrt{\frac{\sum_{i=1}^{n}\left(x_{i}-x_{o}\right)^{2}}{n}},
$$

Table 6 summarizes the statistical results obtained for the predictive performance of the BPNN-Arith. model when applied to the 3D knife form verification samples. In this table, the first column shows the mean and standard deviation (SD) of the evaluation data acquired for each sample in each of the three image perception domains. Meanwhile, the 
second and third columns indicate the Likert scores predicted by the three functional models and the corresponding EI metrics, respectively. Finally, the rightmost column indicates the error rate (ER) values (in percentage terms) for each sample. As shown in Table 6, the average ER values of the "Elegant", "Modern" and "Individualized" prediction models are $18.3 \%, 16.0 \%$ and $19.7 \%$, respectively. The average ER values demonstrate the basic reliability of the functional models in predicting the projected image of the knife form in each of the three image perception domains. Overall, the mean of All-average ER (18.0\%) presented in the lower row of the table, it is inferred that the BPNN model has a better performance for predicting the consumers' likely response to a particular knife form. Therefore, the verification result indicates that the development of a CAPD tool based on the BPNN-Arith. model can not only improve the efficiency of the knife form design task, but also minimize the risk of design decision errors.

Table 6. Manually assigned product image evaluation data, predicted evaluation results, and error rate analysis for BPNN-Arith. prediction model

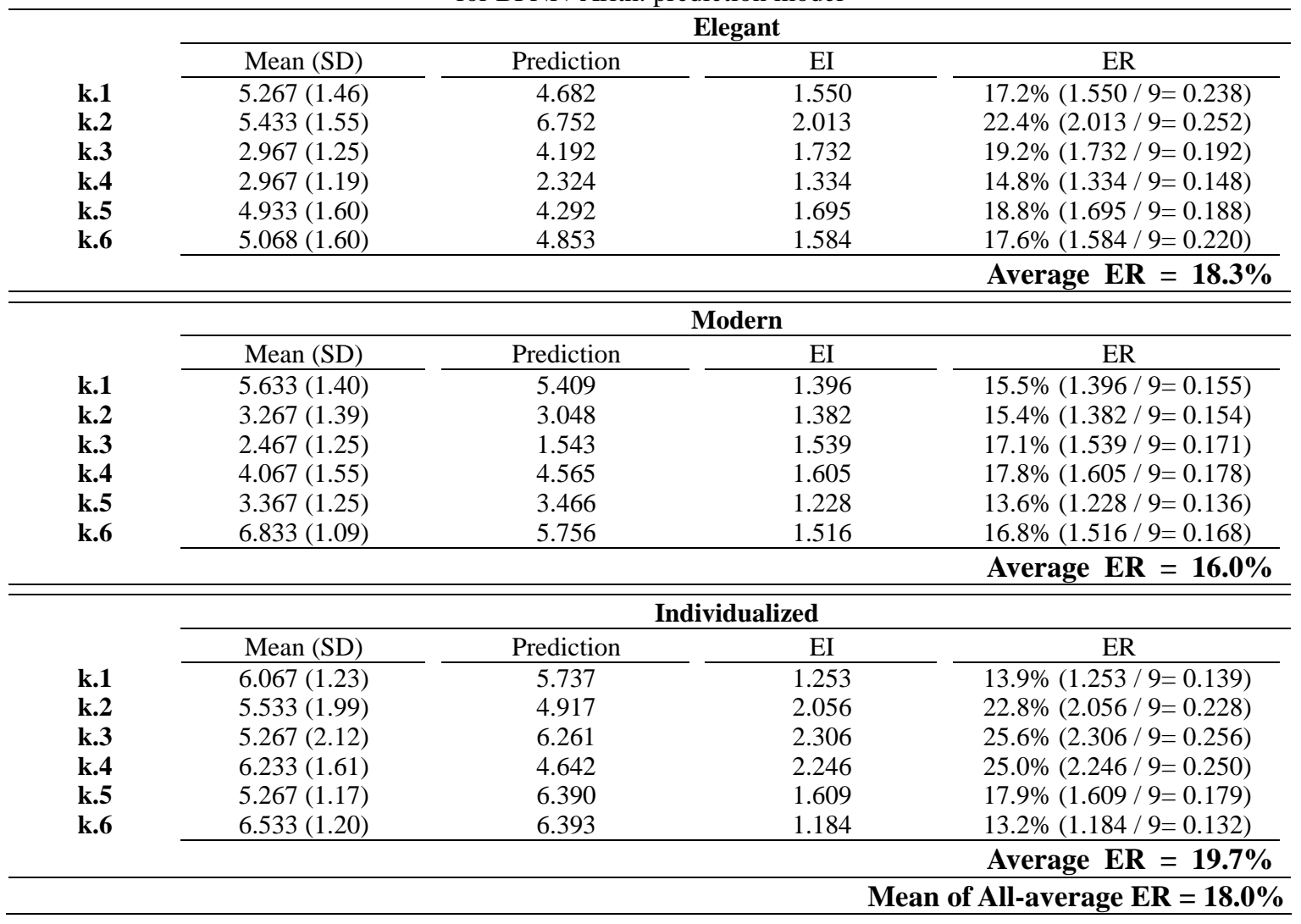

\section{Construction of a CAPD Prototype Tool for Knife Form}

Using Visual Basic software, and taking the trained BPNN-Arith. model described above as its basis, this study developed a computer aided product-form design (CAPD) prototype tool using a knife form for illustration purposes to enable designers to establish consumer image perceptions. Figure 9 shows the interactive interface and the computational flowchart of this prototype tool. After entering the interactive interface, the designer is presented with a default knife form, which he or she can then edit to generate a new knife form by manipulating the values of the thirty-nine design variables specified in the numerical definition of Table 1 and Fig. 3. Having checked the four checkbox items (i.e. "blade upper edge curve", "blade lower edge curve”, "central backbone curve of knife handle", and "tail-section curve") shown in the "Knife-Form Curves Setting" on the upper right side of the interface, pressing the "Edit" button reveals the control points of particular segment of knife profile curves. The designer can then drag the control points to new positions, thereby adjusting these NURBS curves and amending the knife profile accordingly. Having selected any item of "section choice" from a drop-down menu and adjusted each scrollbar horizontal position of four items shown in the "Sectional Detail of Knife Handle", pressing the "Preview" button examines each sectional 
detail variation of knife handle. The designer can then choose the specific item of "section choice" and move each scrollbar to new horizontal position, thereby manipulating the detailed variation of each section of knife handle. Certainly, the manipulation of each design variable on the CAPD tool interface is limited in the specific range as shown in Table 2. Further, having performed the specific settings above, pressing the "Predicting Image" button updates the contents of the "Image Evaluation" boxes on the lower right side of the interface to reveal the predicted image values of the new knife form. Finally, the designer can also press the "Rendering" button rapidly generate a high-lever model of knife from by computation for viewing the visual effect of knife from.

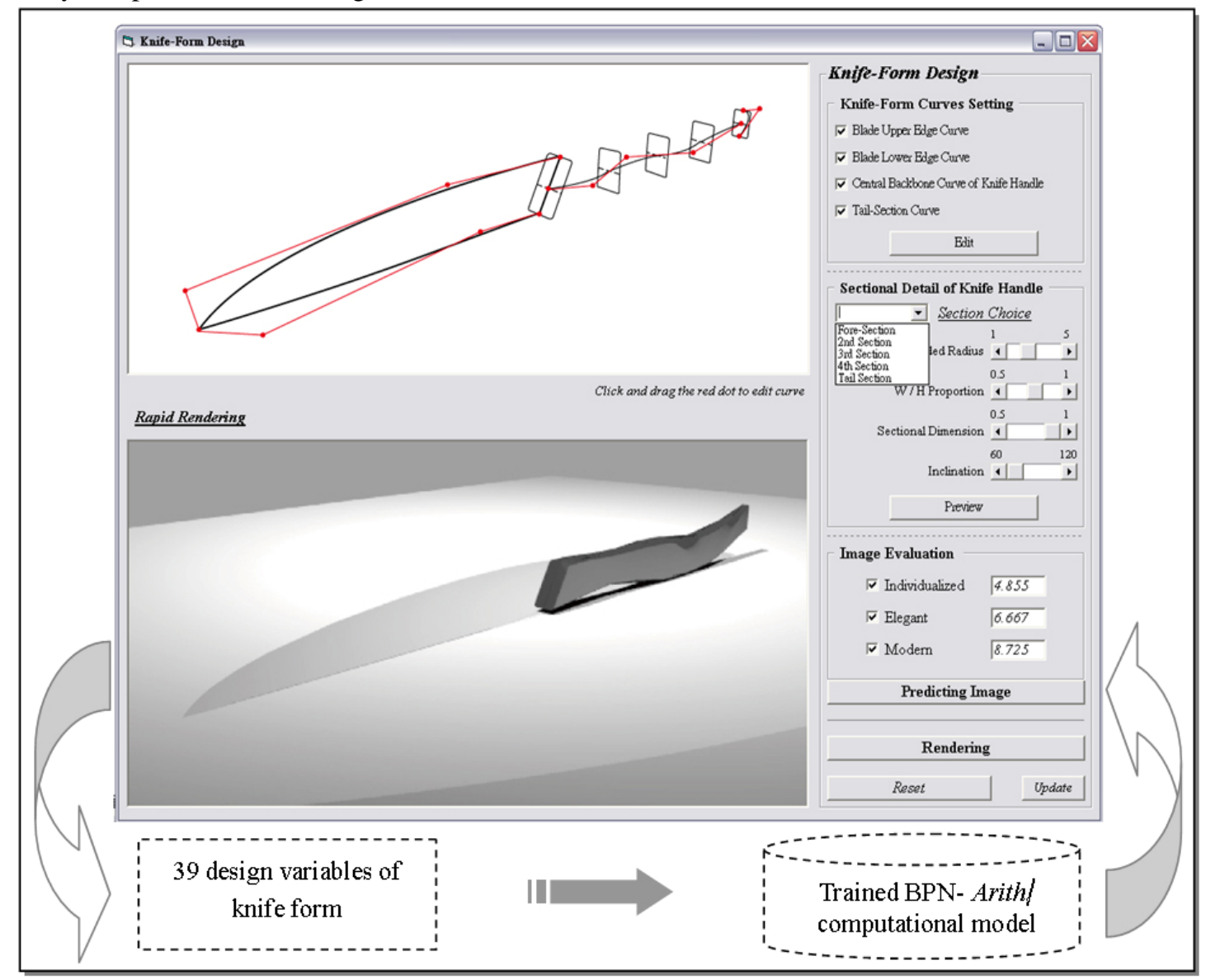

Fig. 9 Interface and computational flowchart of prototype tool

\section{Discussions}

\subsection{Difference between NDS and KE Approach}

In the absence of reliable tools, product designers have little choice but to use intuitive approaches when creating product forms designed to satisfy consumers' product image preferences. Although Kansei Engineering (KE) provides a consumer-orientated design approach for meeting product form expectations, the studies presented in the literature generally employ qualitative techniques to define the overall product form. Consequently, a process of subjective conjecturing must still be applied when evaluating the likely effects on the consumers' emotional response of introducing minor changes in the product form. One of the significant contributions of the NDS presented in this study is the ability it gives to designers to specify the product form in a highly detailed manner. Furthermore, the NDS provides an understanding of how these variables should be manipulated in order to obtain or reinforce the desired product image. By contrast, KE product definition approaches, such as those presented in (Lai et al., 2005; Nagamachi, 2002) are based on a high-level qualitative concept of "items" and "categories. This approach provides product designers with no more than a simple pictorial representation of the product form features with which to explore the consumers' likely emotional response. The current results suggest that compared to such KE approaches, the NDS presented in this study provides a more reliable means of generating product forms "guaranteed" to satisfy a specified set of consumer image expectations. Notwithstanding the NDS has the superiority in describing the detailed product 
form, the NDS still exists a few defects. The main defect is too many variables in describing a product form and leads to the complexity of analytic process. Compared with the simplification of qualitative approach in describing a product form, the NDS is worth being further deliberated and discussed how to simplify the variables in describing a product form so as to apply this concept of NDS to other complicated product form and facilitate the analysis of data set. As a whole, a major difference between the proposed approach of developing a CAPD tool and previous KE approaches lies in their respective objectives. The main purpose of the proposed approach is to construct a mathematical model capable of describing the anticipated consumers' psychological response to a product, whereas the intention of previous KE approaches are to construct the optimal combination of separate product form features to satisfy the consumers' psychological perception of the majority (Lai et al, 2005; Chen and Chuang, 2008; Wang, 2011; Chang and Chen, 2014). Therefore, implementing the proposed approach in the form of a CAPD interface provides the designer with a powerful design tool for evaluating the likely consumer response to detailed changes in the product form definition. Using the CAPD tool, designers can quickly respond to changing consumer requirements by redesigning existing products by adjusting their global or regional form feature variables.

\subsection{Techniques for Constructing Correlation Model}

In this study, the relationship between the independent variables (i.e. the product form details) and the dependent variables (i.e. the consumers' perception of the product image) is established using a BPNN. The BPNN-Arith. model has been adopted as the operational BPNN model for predicting the knife form image values associated with any given numerical knife form definition. The verification result presented in Table 6 has shown that the BPNN model provide a reliable predictive performance. However, BPNN models with their sophisticated nonlinear algorithms are often opaque (Petiota and Yannou, 2004) and it is therefore frequently difficult to recognize the specific design variables which dominate the consumers' response to the product design. By contrast, Multiple Regression Analysis (MRA) or Support Vector Machine Recursive Feature Elimination (SVM-RFE) technique (Shieh and Yang, 2008) allow designers to construct relationship models comprising only those independent variables which exert the most significant effect on the dependent values. Accordingly, it would be worthwhile considering the combination of the variable selection advantage of MRA or SVM-RFE and the sophisticated data analysis capabilities of BPNN, and contemplating the use of alternative methods for establishing the relationship between the dependent variables and the independent variables in the product form design field. For example, using fuzzy rule-based approach (Petiota and Yannou, 2004) to product image descriptors used by consumers when expressing their perceptions of a particular product, or integrating fuzzy algorithm into neural networks to establish the fuzzy neural network model (Hsiao and Tsai, 2005) may provide a more suitable approach than the BPNN technique considered in the present study since such techniques are known to enable highly accurate predictions to be obtained even when the model includes a large number of independent variables.

\section{Conclusion and Future Study}

Consumers' psychological responses to a product form are generally subjective and vague. Designers often tend to be restricted by existing products and by their individual experiences when designing a product form at the conceptual design stage. Therefore, developing computer aided product-form design based on a feasible systematic approach is of crucial importance. Consequently, this study has introduced a systematic approach which uses numerical definition scheme (NDS) and back-propagation neural network (BPNN) to develop a computer aided product-form design (CAPD) tool. The performance of the CAPD tool has been confirmed the accuracy of the image predictions using 6 verification knife forms. Therefore, the CAPD tool provides designers with the ability to predict the likely consumer image perception when presented with a particular knife form. As a result, the designer is in a better position to make an appropriate design decision. Notwithstanding the CAPD tool provides designers with valuable insights into the consumers' visual psychological response induced by a product form, the development of CAPD tool lacks the ergonomic consideration of product form fitness for use. In practice, the ergonomic consideration is also an important perception of consumers about the fitness of use of product forms. It is the characteristic of the product form that can attract consumers at the first instance and consider them for purchase. The perception takes place in the prepurchase context when a consumer may either be consciously or unconsciously looking for a product to buy. Such being the case, it represents a significant determinant in the consumers' selection of products including a product's desirability. 
Since this study mainly focuses on the consumers' visual psychological response to a product form, the ergonomics of product form fitness for use is not considered here. According, a future research should synthetically consider both the range of the ergonomics of product form fitness for use and the consumers' visual psychological response to a product form in order to establish an optimum balance. Finally, although this study has taken the form design of a knife product as a case study, the proposed approach of developing a CAPD tool is also suitable for the design and development of other products.

\section{References}

Chang, H.C. and Chen, H.Y., Optimizing product form attractiveness using Taguchi method and TOPSIS algorithm: a case study involving a passenger car, Concurrent Engineering Research and Applications, 22(2) (2014), pp.135-147

Chang, H.C. Lai, H.H. and Chang, Y.M., 'A measurement scale for evaluating the attractiveness of a passenger car form aimed at young consumers'. International Journal of Industrial Ergonomics, 37 (2007), pp.21-30.

Chang, H.C. Lai, H.H. and Chang, Y.M., 'Expression modes used by consumers in conveying desire for product form: A case study of a car, International Journal of Industrial Ergonomics'. 36 (2006), pp.3-10.

Chen, C. C., Chuang, M. C., Integrating the Kano model into a robust design approach to enhance customer satisfaction with product design. International Journal of Production Economics, 114(2) (2008), 667-681.

Chen, S. E. and Parent, R. E., 'Shape averaging and its applications to industrial design'. IEEE Computer Graphics \& Applications, 9(1) (1989), pp.47-54.

Choi, K. and Jun, C., 'A systematic approach to the Kansei factors of tactile sense regarding the surface roughness'. Applied Ergonomics, 38 (2007), pp.53-63.

Cross, N., 'Engineering design methods: strategies for product design'. John Wiley \& Sons Ltd., Baffins Lane, Chichester (1994).

Demirbilek, O. and Sener, B., 'Product design, semantics and emotional response'. Ergonomics, 46(13/14) (2003), pp.1346-1360.

Green, P. E. and Srinivasan, V., 'Conjoint analysis in consumer research: issues and outlook'. Journal of Consumer Research, 5(1) (1978), pp.103-123.

Han, S. H. and Hong, S. W., 'A systematic approach for coupling user satisfaction with product design'. Ergonomics, 46(13/14) (2003), pp.1441-1461.

Hayashi, C. Method of Quantification, Toyokeizai, Tokyo (1976). (in Japan)

Hsiao, S.W. and Tsai, H.C., 'Applying a hybrid approach based on fuzzy neural network and genetic algorithm to product form design'. International Journal of Industrial Ergonomics, 35 (2005), pp.411-428.

Huang, M.S. Tsai, H.C. and Huang, T.H., 'Applying Kansei engineering to industrial machinery trade show booth design'. International Journal of Industrial Ergonomics, 41 (2011), pp.72-78.

Huang, Y., Chen, C.H. and Khoo, L.P., Products classification in emotional design using a basic-emotion based semantic differential method, International Journal of Industrial Ergonomics 42(6) (2012), pp.569-580.

Jensen, R., The Dream Society: How the Coming Shift from Information to Imagination Will Transform Your Business, New York, McGraw-Hill (1999).

Lai, H. H. Lin, Y. C. and Yeh, C. H., 'Form design of product image using grey relational analysis and neural network models'. Computers \& Operations Research, 32(10) (2005), pp.2689-2711.

Lin, Y.C. Lai, H.H. and Yeh, C.H., 'Consumer oriented design based on fuzzy logic to product form design: a case study of mobile phones'. International Journal of Industrial Ergonomics, 37(6) (2006), pp.531-543.

Llinaresa, C. and Page, A., 'Application of product differential semantics to quantify purchaser perceptions in housing assessment'. Building and Environment, 42 (2007), pp.2488-2497.

$\mathrm{Lu}, \mathrm{X}$. and Zhang, W.J., 'Comparison of different methods for variable selection'. Analytica Chimica Acta, 446(1) (2001), pp.477-483.

McDonagh, D. and Denton, H., 'Exploring the degree to which individual students share a common perception of specific mood boards: observations relating to teaching', learning and team-based design'. Design Studies, 26(1) (2005), pp.35-53. 
Miller, P. H., 'The Magic seven, plus or minus two: Some limit on our capacity to process information'. Psychological Review, 63(1983), pp.81-87.

Nagamachi, M., 'Kansei engineering as a powerful consumer-oriented technology for product development'. Applied Ergonomics, 33 (2002), pp.298-294.

Negnevitsky, M., ArtiIcial intelligence. New York: Addison-Wesley (2002).

Park, J. and Han, S. H., 'A fuzzy rule-based approach to modeling affective user satisfaction towards office chair design'. International Journal of Industrial Ergonomics, 34 (2004)., pp.31-47.

Petiot, J.F. and Yannou, B., 'Measuring consumer perceptions for a better comprehension, specification and assessment of product semantics'. International Journal of Industrial Ergonomics 33 (2004), pp.507-525.

Salhieh, S. M., 'A methodology to redesign heterogeneous product portfolios as homogeneous product families'. Computer-Aided Design, 39 (2007), pp.1065-1074.

Shieh, M. D., Yang, C. C. Multiclass SVM-RFE for product form feature selection. Expert Systems with Applications, 35(1-2) (2008), 531-541.

Smith, S. and Fu, S.H., The relationships between automobile head-up display presentation images and drivers' Kansei', Displays, 32(2011), 58-68.

Van Lottum, C. Pearce, K. and Coleman, S. 'Features of Kansei engineering characterizing its use in two studies: men's everyday footwear and historic footwear'. Quality and Reliability Engineering International, 22 (2006), pp.629-650.

Wang, K.C., A hybrid Kansei engineering design expert system based on grey system theory and support vector regression, Expert Systems with Applications, 38 (2011), pp.8738-8750.

Ward, J. H., 'Hierarchical grouping to optimize an objective function'. Journal of the American Statistical Association, 58 (1963), pp.236-244.

Weiss, D.J., 'Multivariate Procedure'. In: M. D. Dunneltte (Ed), Handbook Industrial and Organizational Psychology. John Willey and Sons, New York(1983), pp.322-362. 